\title{
CONSECUENCIAS DE LA DEGRADACIÓN DE PASTURAS SOBRE LA FIJACIÓN DE NITRÓGENO
}

\section{Consequences of the degradation of pastures on nitrogen fixation}

Julio César Blanco Rodríguez ${ }^{1^{*}}$, Maria Antonia Montilla Rodríguez ${ }^{2}$

Carmen Silvia Roncedo ${ }^{3}$

\section{Artículo de Reflexión}

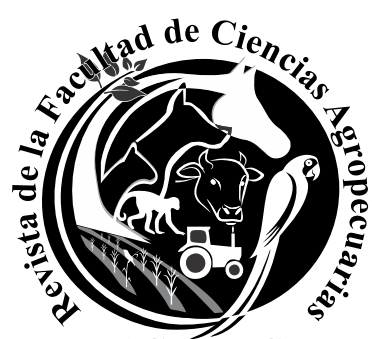

FAGROPEC

Recibido 21 de enero de 2019. Aceptado 15 de marzo de 2019.

${ }^{1}$ Médico Veterinario Zootecnista, Esp, MSc. Profesor Universidad de la Amazonia, Líder Semilleros SIEPSA y SIMCO. Líder grupo GIPSA

(iD) https://orcid.org/0000-0003-0422-8996

${ }^{2}$ Médico Veterinario Zootecnista, MSc (c). Profesor Universidad de la Amazonia. Líder Semillero SIAA. Investigador grupo GIPSA

(iD) https://orcid.org/0000-0001-8092-4390

${ }^{3}$ Ingeniero Zootecnista, Mg. Profesor Universidad Nacional de Tucumán. Facultad de Agronomía y Zootecnia

\section{Como citar:}

BLANCO RODRÍGUEZ, J. C. et al., Consecuencias de la degradación de pasturas sobre la fijación de nitrógeno. En: Revista Facultad Ciencias Agropecuarias FAGROPEC. Universidad de la Amazonia, Florencia - Caquetá. Volumen 11 enero-junio, 2019. Pp. 54-63. ISSN-Revista en Línea: 2539$178 \mathrm{X}$

\section{RESUMEN}

La degradación de pasturas es un problema de interés mundial que afecta la producción y la salud ecosistémica. Cada estado de degradación de pasturas, corresponde a un avance gradual en el deterioro de la relación entre el suelo, la planta, el bovino y el medio ambiente, convirtiendo la ganadería poco a poco en un foco de contaminación. Una de esas fuentes contaminantes corresponde a los GEI (Gases Efecto Invernadero) de los cuales, $\mathrm{N}_{2} \mathrm{O}$ es el que presenta mayor potencial de calentamiento global; por esta razón, en el presente artículo se relacionan generalidades de la degradación de pasturas y su relación con la emisión de gases nitrogenados, entre los cuales se incluye el $\mathrm{NH}_{3}, \mathrm{~N}_{2} \mathrm{O}$ y NO , a través de una revisión de documentos resultantes de diferentes investigaciones a nivel mundial, enfocados en la experiencia de los autores.

Palabras claves:

Degradación; Gases; Invernadero; Fijación de nitrógeno

\begin{abstract}
Pasture degradation is a problem of global interest that affects production and ecosystem health. Each state of pasture degradation corresponds to a gradual advance in the soil, plant, bovine and the environmental deterioration, gradually turning livestock farming into a source of contamination. One of these polluting sources corresponds to the GHG (Greenhouse Gases) of which, $\mathrm{N}_{2} \mathrm{O}$ is the one with the greatest potential for global warming; For this reason, this article relates generalities of pasture degradation to its relationship with the emission of nitrogen gases, including $\mathrm{NH}_{3}, \mathrm{~N}_{2} \mathrm{O}$ and $\mathrm{NO}$, through the review of documents of different worldwide investigations, focused on the authors' experience.
\end{abstract}

Key words:

Degradation; Gases; Greenhouse; nitrogen fixation

*Autor para correspondencia: mvzjulioblanco@gmail.com 


\section{INTRODUCCIÓN}

Una de las relaciones intrínsecas en los ecosistemas de pastura, corresponde al ciclo del N (servicio ecosistémico) y sus efectos frente a los valores de proteína (bien) en los subproductos de origen animal. Este aspecto hace que en ganadería el $\mathrm{N}$ sea considerado como el elemento más importante para el desarrollo de la vegetación después del agua y supone entre 1,5 y el 5\% de la planta, por lo cual su deficiencia en los suelos hace de él uno de los elementos clave a nivel mineral para la producción animal(Escaso, Martínez y Planelló 2010).

Por tal razón, el metabolismo de $\mathrm{N}$ es un factor limitante en la síntesis de proteínas cuya cantidad y calidad presente en las plantas, lo animales o productos de estos, es decisiva para determinar su utilidad como alimento (Castillo, Vecino U y Dixon 2011), ya que depende de la reducción del $\mathrm{N}_{2}$ (nitrógeno atmosférico) por parte de microorganismos asociativos y su presencia en las plantas huésped y demás forrajeras utilizadas por los animales de pastoreo (Dubeux Jr. et al. 2014), que son empleadas por los microorganismos ruminales que sintetizan el nitrógeno y lo reciclan nuevamente hacia el rumen a través de la saliva. Posteriormente, la proteína degradable obenida de los forrajes se metaboliza en el hígado a partir de aminoácidos que harán parte del tejido animal y la proteína de la leche (Church 1988), lo que determina la calidad del producto final que consume la población humana.

La necesidad de los microorganismos en la mayor parte del $\mathrm{N}$ se encuentra en el suelo como $\mathrm{N}$ orgánico (aminas, amidas, etc.), razón por la que la planta no puede absorberlo. De esta manera son importantes los procesos de mineralización que generalmente se regulan por microorganismos (fijación biológica de nitrógeno), cuya sucesión impacta sobre la fertilidad(Li etal. 2019) y por ende sobre la producción animal, lo que sustenta la importancia de comprender la relación entre el estado de las pasturas y la fijación de N.

Por lo anterior, el objetivo del presente artículo, es exteriorizar características generales de la fijación biológica de nitrógeno y su relación con las emisiones de GEI que pueden alterarse como consecuencia de la degradación de las pasturas.

\section{METODOLOGÍA}

Se llevó a cabo una investigación metódica de documentos resultado de investigación, relacionados con degradación de pasturas, fijación biológica de nitrógeno y emisiones nitrogenadas de GEI.

Como producto de la búsqueda de información, se seleccionaron 100 documentos relacionados con libros, tesis y artículos científicos en diferentes idiomas, utilizando las plataformas Scopus, Elsevier, Springer, Scielo, Redalyc, Google y Google Académico.

A partir de las publicaciones científicas revisadas, se encontró que 60 de ellas no fueron relevantes con el objetivo del producto esperado, por lo cual fueron excluídas, ya que el estudio buscó contrastar el conocimiento de los autores con las particularidades del tema, cuya importancia actual es notable, dada la repulsión que genera la producción bovina ante los movimientos ambientalistas mundiales, teniendo en cuenta la relación directa que existe entre esta actividad y la emisión de GEI; al igual que, 
la correspondencia entre el establecimiento de pasturas y la deforestación, más aún cuando la degradación de pasturas se convierte en un generador de la eliminación de áreas de bosque.

Igualmente, se compararon otros autores como Barcellos (1986), Spain \& Gualdrón (1991), Andrade- Lira, et al (2006), Blanco- Rodríguez (2010), Passos, et al (2015), Yao, et al (2016), entre otros, quienes han generado diferentes clasificaciones de estados de degradación, a fin de evidenciar si dichas categorías están asociadas con rangos o valores de emisiones de GEI nitrogenadas producto de las alteraciones de la fijación biológica de nitrógeno.

\section{RESULTADOS Y DISCUSIÓN}

\section{Degradación de pasturas}

Inicialmente, la degradación de pasturas se consideró por Spain \& Gualdrón (1991), como una disminución en la productividad a causa de condiciones climáticas y edáficas a las que las mismas está siendo sometida. Sin embargo, autores como Según Macedo (2000), (Betancourt 2006), Escobar, et al (2001), Dias-Filho (2007) y Padilla, et al (2013) indican que la degradación de pasturas es una pérdida de productividad y de capacidad de recuperación natural de las plantas cultivadas para las actividades de pastoreo, que se ven reflejadas en una mala calidad de la cobertura con imposibilidad para sostener animales (capacidad de carga) y problemas posteriores como disminución notable de biomasa aérea, compactación y erosión, afectando los ciclos biogeoquímicos que se refleja en indicadores de fertilidad del suelo.

Lo anerior se sustenta lo obtenido por Ramírez y Seré (1990) quienes desarrollan un análisis sobre la implementación de Brachiaria decumbens en ganaderías de doble propósito en Caquetá y consideran que la infertilidad es una consecuencia de la degradación de pasturas al igual que la disminución en el nivel de proteína del forraje, cobertura de la gramínea, suelo descubierto y nivel de incidencia de salivazo. Sin embargo, Miles, et al (1998), afirman que la baja fertilidad del suelo es una causa de la degradación de pasturas mas no una consecuencia, peroEscobar et al (2001) aseveran que la infertilidad de los suelos es una consecuencia de la degradación de las pasturas por un sistema inadecuado de manejo lo cual coincide con los planteamientos de Ramírez y Seré (1990), Motta Macedo (2000), Betancourt, (2006), Escobar, et al (2001), Dias- Filho (2007), Padilla et al. (2013) y Yao, et al. (2016).

La degradación de pasturas es considerada un problema mundial (Holzner y Kriechbaum, 2001), que se atribuye a los pastos mejorados (Padilla et al, 2013). Sin embargo, Dias-Filho (2007) afirma que este inconveniente afecta principalmente las pasturas formadas en los diferentes ecosistemas de América Latina tropical, por lo cual se conocen avances al respecto en países como, Costa Rica (Betancourt 2006), Argentina (Roncedo 2007) y principalmente Brasil, donde autores como Nascimento Junior \& Almeida, 2001), Serrao y Toledo (1990), Macedo y Zimmer (2000), Bonatto et al (2007) y Dias-Filho (2007) entre otros, donde se ha estudiado la degradación como un problema productivo con repercusiones ambientales.

Miles, et al (1998) Indicó que existe bastante información de tipo anecdótica sobre la degradación de pasturas, pero escasos datos científicos y afirman que se dispone de poca información sobre investigaciones referentes a pasturas de Brachiaria degradadas durante periodos largos, deduciendo 
que se necesitan más estudios a cerca de procesos de degradación.

\section{Fijación de nitrógeno en pasturas degradadas}

La fijación de nitrógeno en las pasturas se puede dar de dos formas: la primera, mediante bacterias asociadas a especies de leguminosas (fijación simbiótica) y la segunda, a través de bacterias de vida libre (fijación asimbiótica) que habitan la rizosfera(Moreno y Galvis 2018)(Moreno y Galvis 2018). Sin embargo, puede existir otro tipo de fijación a través de las excresiones (heces y orina) que también incorporan el material consumido al suelo, tal y como lo afirman Hakala \& Jauhiainen (2007)y Dubeux, et al (2014).

La fijación simbiótica se da a causa de la ausencia de interacciones microbianas al interior de las plantas, situación que las obliga a desarrollar rasgos para estimular y manipular las relaciones con los microorganismos ( Revillini et al., 2016). De esta forma, la planta excreta a través de las raíces ciertos factores de crecimiento (Devlin, 1982) o exudados que son detectados por las bacterias a través de quimiotaxis y les permite atravesar la punta de las vellosidades radicales, formando una curvatura o rizado(Garg y Renseigné 2007). Esto hace que cada bacteria atrapada en la bolsa del rizo invada vellosidades dañadas o rotas y puedan desarrollarse a lo largo de un filamento de infección en el tejido cortical hasta llegar al área inmediata de la endodermis (capa de células de la raíz) y al prericiclo (tejido que rodea al cilindro vascular de la raíz) donde se producen divisiones celulares y el nódulo crece rápidamente, aumentando el volumen en dirección a la superficie de la raíz.

Esta actividada microbiológica, permite que el nitrógeno atmosférico o $\mathrm{N}_{2}$ se reduzca a formas reactivas y biológicamente disponibles, pero su aprovechamiento por parte de las especies vegetales depende también de las condiciones de la pastura misma, de tal manera que factores como la humedad(Pajares y Bohannan, 2016) u otras condiciones ecológicas que determinan el tipo de suelo, pueden inhibir la actividad de la enzima nitrogenasa(Stone, Kan y Plante 2015) afectando la fijación biológica de nitrógeno. Por tal razón, las pasturas con avanzado estado de degradación, que presentan suelo compactado, manifiestan altas perdidas de nitrógeno(Dias-Filho 2015).

Tal y como se observa en la figura 1, el nitrógeno es fijado al suelo en forma de $\mathrm{NH}_{4}$ el cual luego es convertido en Nitrito $\left(\mathrm{NO}_{2}\right)$ y posteriormente a $\mathrm{NO}_{3}$. Sin embargo, el $\mathrm{NO}_{3}$ que no puede lixiviarse o absorberse, transformándose en $\mathrm{N}_{2}$ a través de la desnitrificación por medio de bacterias del género Nitrosoma, Pseudomona y Nitrobacter, generando $\mathrm{N}_{2} \mathrm{O}$ como parte de un proceso químico obligado (Garzón y Cárdenas 2013). Estos se incorporan en la atmósfera como parte del ciclo natural. Es así, que gases como $\mathrm{NH}_{3}, \mathrm{~N}_{2} \mathrm{O}$ y NO, son emitidos a la atmósfera, de forma normal desde las pasturas como parte del ciclo del nitrógeno, considerados gases contaminantes de la atmósfera, pero en condiciones ideales, las cantidades emitidas son catalizadas en la estratósfera por procesos de fotodescomposición obteniéndose $\mathrm{H}, \mathrm{O}_{2}, \mathrm{~N}_{2}, \mathrm{O}_{3}$ (Moreno 2016).

Teniendo en cuenta que la degradación de las pasturas no permite las condiciones ideales de emisión a la atmósfera, al convertirse en fuentes potenciales de $\mathrm{N}_{2} \mathrm{O}$ (Dias-Filho 2015), es necesario que el ganadero reconozca que uno de sus aliados son las leguminosas, ya que al ser especies estrechamente relacionadas con la fijación biológica de nitrógeno, son importantes para la mitigación de emisiones de gases efecto invernadero (GEI) nitrogenados, pues su participación anual está entre 33 a $46 \mathrm{Tg}$ (Teragramos) fijados de $\mathrm{N}_{2}$ en el suelo, cantidad que posteriormente es reducida, asimilada por las 


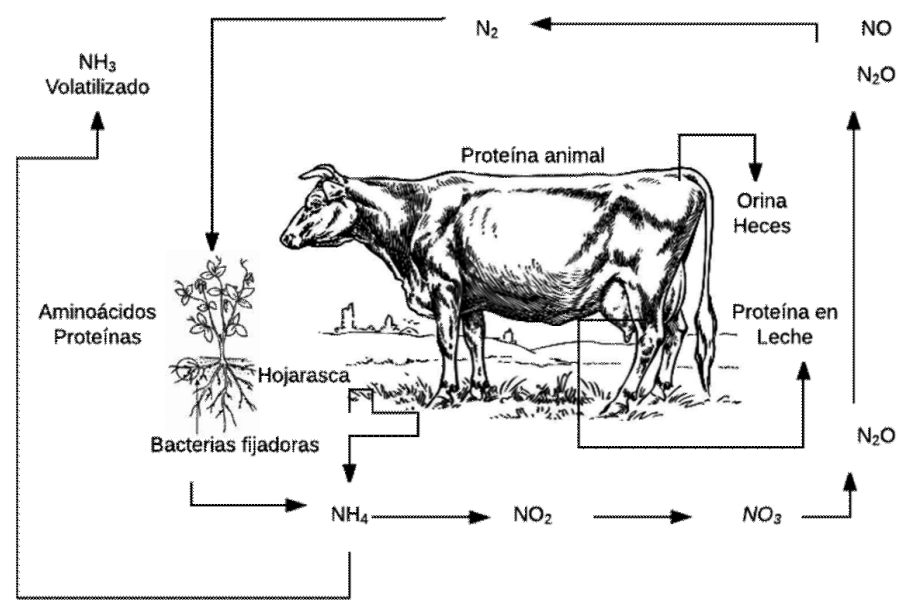

Figura 1. Ciclo de nitrógeno en pasturas.

plantas y transformada en compuestos orgánicos más complejos tales como aminoácidos y proteínas que serán consumidas en las pasturas donde se ha fijado(Jensen et al. 2012).

Igualmente, la pastura que se encuentra en estado de degradación avanzado y no cuenta con una presencia adecuada de leguminosas que fijen el nitrógeno en el suelo (Macharia etal. 2011), verá afectada la calidad nutricional de los forrajes y la carga animal, al igual que la cantidad y calidad de la leche producida. Esto se sustenta a través de autores como Njarui, et al (2010) quienes indican que la asociación de gramíneas con leguminosas es importante para la producción de biomasa y calidad de la pastura o de investigadores como Cipagauta, Velásquez y Pulido (1998), que determinan incrementos en la producción de leche de hasta en $23 \%$ cuando hay leguminosas en la pastura, al ser comparadas con monocultivo de gramíneas.

Cabe aclarar, que las pasturas no están exentas de un proceso de degradación al tener leguminosas dentro de su cobertura, esto indica que el mal manejo no distingue especie vegetal. Es por esto, que una pastura degradada pierde hasta un $78 \%$ de la composición vegetal, lo que dificulta la fijación biológica de nitrógeno, afectando la producción y la salud ambiental.

\section{Emisiones antropogénicas nitrogenadas en pasturas degradadas}

Cuando se presenta el proceso de degradación de las pasturas, las condiciones se alteran por completo, por una pérdida de productividad, que según Macedo (2000), puede evidenciarse por la disminución de la capacidad de recuperación natural de la pastura para sustentar los niveles de producción exigidos por los animales (capacidad de carga). Esta condición permite que las especies vegetales asociadas con la fijación biológica de $\mathrm{N}_{2}$ se conviertan en un elemento importante dentro de la cobertura vegetal de la pastura para su recuperación, ya que Phelan et al (2014) afirman que al presentar simbiosis con bacterias fijadoras de $\mathrm{N}$, puede aumentarse la capacidad de producción de forraje, su calidad y por ende la producción animal, particularmente en áreas donde no se utilizan fertilizantes.

Sin embargo, en campo la realidad es otra, debido a que al degradarse la pastura la presión por pastoreo aumenta y se generan procesos de compactación del suelo, a causa de un pastoreo selectivo 
con interferencia del ciclo del N (fijación biológica), que se genera al haber deposición irregular de heces y orina lo que incide directamente en agravar el proceso de degradación (Dias-Filho, 2007), ya que contienen cantidades importantes del elemento.

Saggar, et al (2013) afirman que aproximadamente 60 a 75\% del N presente en la dieta se excreta en la orina y Pedraza (1986), indica que el $\mathrm{N}$ del estiércol corresponde en promedio al 17,8\% de la materia seca ingerida por vacas lecheras. Así mismo, en pasturas activas, la alta deposición de orina y heces, particularmente en las áreas de descanso, bebederos, lugares de suministro de sales mineralizadas y caminos creados por las vacas, genera importantes pérdidas de nitrógeno debido a que estas superficies no poseen cobertura vegetal, al igual que sucede con extensiones de pastura que presentan altos niveles de compactación como consecuencia de un proceso avanzado de degradación (DiasFilho, 2007)

Por otra parte, los ganaderos al desconocer la importancia de la fijación biológica de $\mathrm{N}_{2}$ en las pasturas, se limitan a fertilizar inadecuadamente en algunos casos donde las condiciones económicas lo permiten omitiendo si es necesario o no hacerlo.

Esta situación aumenta la volatilización de $\mathrm{NH}_{3}$ a la atmósfera como resultado de altas entradas de nitrógeno al ecosistema de pasturas, incrementando los niveles atmosféricos de $\mathrm{N}_{2} 0$ y $\mathrm{NO}$ ya que es un predecesor de estos (Garzón \& Cárdenas, 2013). El $\mathrm{N}_{2} \mathrm{O}$ es un gas efecto invernadero bastante poderoso (Barneize et al, 2014) con un potencial de calentamiento global 298 veces mayor a la del dióxido de carbono (Solomon y Qin 2013). El NO es un gas tóxico, bastante estudiado por la medicina, debido a que regula funciones celulares como es el caso de la presión sanguínea (Centelles, Esteban y Imperial 2004), la hemodinámica vascular y algunas funciones hematológicas y de defensa (Ferrer-Viant et al, 1998). Por lo anterior, las actividades inadecuadas de producción y el mal uso de las pasturas son actualmente un motor de deterioro de la humanidad.

\section{CONCLUSIONES}

El nitrógeno como componente principal de las proteínas de las células es esencial en las pasturas para la alimentación bovina, pues se considera uno de los elementos principales de la dieta y de mayor costo; razón por la cual, la proteína de la leche como producto resultante, posee un incentivo importante en el mercado por parte de la agroindustria.

Pese a lo anterior, la gestión inadecuada de pasturas, conduce a un desbalance en el ciclo de este componente, generando emisiones de amoniaco $\left(\mathrm{NH}_{3}\right)$, óxido nitroso $\left(\mathrm{N}_{2} \mathrm{O}\right)$ y óxido nítrico $(\mathrm{NO})$, considerados gases contaminantes de la atmósfera, que se incrementan por la fertilización inadecuada en la producción ganadera y la alta deposición de orina y heces en áreas desnudas o compactadas por pastoreo selectivo, como consecuencia de la degradación de pasturas.

Finalmente, cabe resaltar que frente a la relación entre degradación de pasturas, fijación biológica de nitrógeno y sus efectos en las emisiones de GEI, no se evidenciaron datos que permitan indicar que existan investigaciones a través de las cuales se analicen valores de gases nitrogenados, producto del ciclo del nitrógeno en pasturas degradadas, razón por la cual, es necesario desarrollar estudios que evidencien detalladamente como este fenómeno causado por el mal manejo, afecta la salud 
ambiental.

\section{LITERATURA CITADA}

ANDRADE- LIRA, M.D.E., VIRGINIA, M., DOS, F. y CARLOS, J., 2006. Sistemas de produção de forragem: alternativas para sustentabilidade da pecuária. 43a Runiao anual da SBZ- Joao Pessoa - PB. S.1.: Revista Brasileira de Zootecnia, pp. 405-423.

BARCELLOS, A.., 1986. Recuperacao de pastagens degradadas. 1986. Planaltina, Brasil: EMBRAPA y CPAC.

BARNEZE, A.S., MAZZETTO, A.M., ZANI, C.F., MISSELBROOK, T. y CERRI, C.C., 2014. Nitrous oxide emissions from soil due to urine deposition by grazing cattle in Brazil. Atmospheric Environment [en línea], vol. 92, pp. 394-397. ISSN 18732844. DOI 10.1016/j.atmosenv.2014.04.046. Disponible en: http://dx.doi.org/10.1016/j.atmosenv.2014.04.046.

BETANCOURT, H., 2006. Evaluación bioeconómica del impacto de la degradación de pasturas en fincas ganaderas de doble propósito en El Chal, Petén, Guatemala [en línea]. S.l.: CATIE. Disponible en: http://orton.catie.ac.cr/repdoc/A2242E/A2242E.PDF.

BLANCO- RODRÍGUEZ, J.., 2010. Caracterización de estados de degradación de pasturas, definidos a partir de índices de vegetación. S.l.: Universidad Nacional de Colombia - Universidad de la Amazonia.

BLANCO-RODRÍGUEZ, JULIO CÉSAR; RODRÍGUEZ, L. C.H; MALAGÓN, R; TORRES, M.N.., 2010. Characteristics recognition of pastures degradation at San Luis Farm Morelia- Caquetá - Colombia. Revista Facultad de Ciencias Agropecuarias [en línea], vol. 2, no. Enero-Junio 2010, pp. 3-12. Disponible en: http://www.udla.edu.co/revistas/index.php/ciencias-agropecuarias.

CASTILLO, R., VECINO U, C. y DIXON, Y., 2011. Leguminosas nativas en áreas ganaderas de la isla de la juventud, Cuba. Avances en investigación agropecuaria, vol. 15, no. 2, pp. 13-27.

CENTELLES, J.J., ESTEBAN, C. y IMPERIAL, Y.S., 2004. Óxido nítrico. Ambito farmacéutico, vol. 23, pp. 96-102.

CHURCH, D.., 1988. El rumiante. Fisiología digestiva y nutrición. Saragoza, España: Acribia.

CIPAGAUTA, M., VELÁSQUEZ, J. y PULIDO, J.., 1998. Cipagauta et al, 1998.pdf. Pasturas Tropicales, vol. 20, no. 3, pp. 2-10.

DIAS-FILHO, MOACYR, B., 2007. DEGRADACAO DE PASTAGENS procesos, causas e estrategias de recuperacao. Terceira e. Belém do Pará: EMBRAPA. ISBN 85-87690-65-4.

DIAS-FILHO, M.., 2015. DEGRADACAO DE PASTAGENS Processos, Causas e Estratégias de Recuperacao [en línea]. Quarta edi. Belém do Pará: s.n. ISBN 978-85-911831-0-4. Disponible en: www.mbdf.com.gr.

DUBEUX JR., J.C.B., SOLLENBERGER, L.E., SILVA, H.M.S., SOUZA, T.C. de, MOZLEY III, E.L. y SANTOS, E.R.S., 2014. Nutrient Cycling in Tropical Pastures : What do we know ? Conference Paper, no. March 2016. 
ESCASO, S.., MARTÍNEZ, G.J.. y PLANELLÓ, C.M.., 2010. Fundamentos básicos de fisiología vegetal y animal. Madrid-España: Pearson education.

ESCOBAR, M.., ESCOBAR, G. y RIPPSTEIN, G., 2001. Degradación de pasturas mejoradas por la presencia de especies no deseadas en Carimagua, Colombia. En: G. RIPPSTEIN, G. ESCOBAR y F. MOTTA (eds.), Agroecología y Biodiversidad de las Sabanas en los Llanos Orientales de Colombia. 1. Cali, Colombia: CIAT, pp. 308. ISBN 9586940330.

FERRER-VIANT, D., JORGE-FONSECA, C., GARCÍA-RODRÍGUEZ, R., MARTÍNEZ-ANGLADA, P.F., 1998. Óxido nítrico. Importancia Biológica y participación en algunas funciones cardiovasculares y heatológicas. Medisan, vol. 2, no. 1, pp. 45-53.

GARG, N. y RENSEIGNÉ, N., 2007. Symbiotic nitrogen fixation in legume nodules : process and signaling . A review. Agron. Sustain, vol. 27, pp. 59-68. DOI http://dx.doi.org/10.1051/agro:2006030.

GARZÓN, J.E. y CÁRDENAS, E.A., 2013. Emisiones Antropogénicas De Amoniaco , Nitratos Y Óxido Nitroso : Compuestos Nitrogenados Que Afectan El Medio Ambiente En El Sector Agropecuario Colombiano Anthropogenic Emissions of Ammonia, Nitrates and Nitrous Oxide : Nitrogen Compounds That Affect T. Rev. Med. Vet. Zoot., vol. 60, no. Ii, pp. 121-138.

HAKALA, K. y JAUHIAINEN, L., 2007. Yield and nitrogen concentration of above- and below-ground biomasses of red clover cultivars in pure stands and in mixtures with three grass species in northern Europe. Grass and Forage Science, vol. 62, no. 3, pp. 312-321. ISSN 01425242. DOI 10.1111/j.13652494.2007.00585.x.

JENSEN, E.S., PEOPLES, M.B., BODDEY, R.M., GRESSHOFF, P.M., HENRIK, H.N., ALVES, B.J.R. y MORRISON, M.J., 2012. Legumes for mitigation of climate change and the provision of feedstock for biofuels and biorefineries. A review. S.1.: s.n. ISBN 1359301100.

LI, W., LIU, M., WU, M., JIANG, C., KUZYAKOV, Y., GAVRICHKOVA, O., FENG, Y., DONG, Y. y LI, Z., 2019. Bacterial community succession in paddy soil depending on rice fertilization. Applied Soil Ecology [en línea], vol. 144, no. 71, pp. 92-97. ISSN 09291393. DOI 10.1016/j.apsoil.2019.07.014. Disponible en: https://doi.org/10.1016/j.apsoil.2019.07.014.

MACHARIA, P.N., GACHENE, C.K.K., MUREITHI, J.G., KINYAMARIO, J.I., EKAYA, W.N. y THURANIRA, E.G., 2011. The effect of introduced forage legumes on improvement of soil fertility in natural pastures of semi-arid rangelands of Kajiado District, Kenya. Tropical and Subtropical Agroecosystems [en línea], vol. 14, no. 1, pp. 221-227. ISSN 18700462. Disponible en: http://www.redalyc.org/ articulo.oa? $\mathrm{id}=93915703021$.

MILES, J.W., MAASS, B.L. Y DO VALLE, C.B., 1998. Brachiaria: biología, agronomía y mejoramiento. S.l.: s.n.

MORENO, L.. y GALVIS, F., 2018. Potencial biofertilizante de bacterias diazótrofas aisladas de muestras de suelo rizosférico. Pastos y Forrajes, vol. 36, no. 1, pp. 33-37. ISSN 0864-0394.

MORENO, S.A., 2016. Química atmosférica de compuestos orgánicos volátiles oxigenados [en línea]. S.1.: Universidad de Castilla - La Mancha. Disponible en: https://ruidera.uclm.es/xmlui/bitstream/ handle/10578/10712/TESIS Moreno Sanroma.pdf? sequence=1\&isAllowed=y. 
MOTTA MACEDO, M.C., 2000. A Integracao lavoura e pecuaria como alternativa de recuperacao de pastagens degradadas. En: EMBRAPA (ed.), Nitrogenio na sustentabilidade de sistemas intensivos de producao agropecuaria. 1. Dourados: s.n., pp. 163.

NASCIMENTO JUNIOR; ALMEIDA, R., 2001. Degradação , recuperação e sustentabilidade de pastagens cultivadas Degradação, recuperação e sustentabilidade de pastagens. , pp. 1-17.

NJARUI, D.M.., NJOKA, E.., ABDULRAZAK, S.. y MUREITHI, J.., 2010. Efect of planting pattern of two herbaceous forage legumes in fodder grasses on productivity of grass/legume mixture in semi-arid tropical Kenya. Tropical and suptropical agroecosystems, vol. 7, pp. 73-85. DOI 10.4090/juee.2008.v2n2.033040.

PADILLA, C., SARDIÑAS, Y., FEBLES, G. y FRAGA, N., 2013. Estrategias para el control de la degradación en pastizales invadidos por Sporobolus indicus (L) R. Br. Revista Cubana de Ciencia Agrícola, vol. 47, no. 2, pp. 113-117.

PAJARES, S. y BOHANNAN, B.J.M., 2016. Ecology of Nitrogen Fixing , Nitrifying, and Denitrifying Microorganisms in Tropical Forest Soils. , vol. 7, no. July, pp. 1-20. DOI 10.3389/fmicb.2016.01045.

PASSOS, R.R., COSTA, L.M., BURAK, D.L. y SANTOS, D.A., 2015. Quality indices in degraded pasture in hilly relief. Semina:Ciencias Agrarias, vol. 36, no. 4, pp. 2465-2482. ISSN 16790359. DOI 10.5433/16790359.2015v36n4p2465.

PEDRAZA, C., 1986. Manejo y propiedades del estiércol de lechería. IPA La Platina, no. 38, pp. 56-60.

PHELAN, P., MOLONEY, P., MCGEOUGH, E.., HUMPHREYS, J., BERTILSSON, J., O'RIORDAN, E.. y O'KLIELY, P.., 2014. Forage Legumes for Grazing and Conserving in Ruminant Production Systems. Plant Sciences, vol. 34, no. October 29, pp. 282-315. DOI 10.1080/07352689.2014.898455.

REVILLINI, D., GEHRING, C.A., JOHNSON, N.C., REVILLINI, D., GEHRING, C.A. y JOHNSON, N.C., 2016. The role of locally adapted mycorrhizas and rhizobacteria in plant-soil feedback systems The role of locally adapted mycorrhizas and rhizobacteria in plant-soil feedback systems. Functional ecology, no. August, pp. 1-13. DOI 10.1111/1365-2435.12668.

RONCEDO, C.S.; H.E.P.C., 2007. Metodología para evaluar pasturas tropicales degradadas: Chloris Gayana Kunth CV. Común en la llanura deprimida de Tucumá, Argentina. . Leales, Tucumán:

SAGGAR, S., JHA, N., DESLIPPE, J., BOLAN, N.S., LUO, J., GILTRAP, D.L., KIM, D.G., ZAMAN, M. y TILLMAN, R.W., 2013. Denitrification and N2O: N2 production in temperate grasslands: Processes, measurements, modelling and mitigating negative impacts. Science of the Total Environment [en línea], vol. 465, pp. 173-195. ISSN 00489697. DOI 10.1016/j.scitotenv.2012.11.050. Disponible en: http://dx.doi.org/10.1016/j.scitotenv.2012.11.050.

SOLOMON, S. y QIN, D., 2013. Climate Change 2007 The Physical Science Basis The. En: C. UNIVERSITY (ed.), Journal of Chemical Information and Modeling. 1. New York: Friesens, pp. 1689-1699. ISBN 9788578110796.

SPAIN, J.M. y GUALDRÓN, R., 1991. Degradación y rehabilitación de pasturas. Establecimiento y renovacion de pasturas : conceptos, experiencias y enfoques de la investigacion. primera ed. Veracruz, 
México: CIAT (Centro Internacional de Agricultura Tropical), pp. 269-283. ISBN 9589183263.

STONE, M.M., KAN, J. y PLANTE, A.F., 2015. Parent material and vegetation influence bacterial community structure and nitrogen functional genes along deep tropical soil profiles at the Luquillo Critical Zone Observatory. Soil Biology and Biochemistry [en línea], vol. 80, no. 2015, pp. 273-282. ISSN 00380717. DOI 10.1016/j.soilbio.2014.10.019. Disponible en: www.elsevier.com/locate/soilbio\%0AParent.

YAO, Z., ZHAO, C., YANG, K., LIU, W., LI, Y., YOU, J. y XIAO, J., 2016. Alpine grassland degradation in the Qilian Mountains, China - A case study in Damaying Grassland. Catena [en línea], vol. 137, pp. 494-500. IS S N 03418162 . DO I $10.1016 / \mathrm{j}$. catena.2015.09.021. Disponible en: http://dx.doi.org/10.1016/j.catena.2015.09.021. 